\title{
Application of Self-Organizing Artificial Neural Networks on Simulated Diffusion Tensor Images
}

\author{
Dilek Göksel-Duru and Mehmed Özkan \\ Institute of Biomedical Engineering, Bogazici University, Kandilli Campus, 34684 Istanbul, Turkey \\ Correspondence should be addressed to Dilek Göksel-Duru; gokseld@boun.edu.tr
}

Received 4 February 2013; Accepted 18 March 2013

Academic Editor: Matjaz Perc

Copyright (C) 2013 D. Göksel-Duru and M. Özkan. This is an open access article distributed under the Creative Commons Attribution License, which permits unrestricted use, distribution, and reproduction in any medium, provided the original work is properly cited.

\begin{abstract}
Diffusion tensor magnetic resonance imaging (DTMRI) as a noninvasive modality providing in vivo anatomical information allows determination of fiber connections which leads to brain mapping. The success of DTMRI is very much algorithm dependent, and its verification is of great importance due to limited availability of a gold standard in the literature. In this study, unsupervised artificial neural network class, namely, self-organizing maps, is employed to discover the underlying fiber tracts. A common artificial diffusion tensor resource, named "phantom images for simulating tractography errors" (PISTE), is used for the accuracy verification and acceptability of the proposed approach. Four different tract geometries with varying SNRs and fractional anisotropy are investigated. The proposed method, SOFMAT, is able to define the predetermined fiber paths successfully with a standard deviation of $(0.8-1.9) \times 10^{-3}$ depending on the trajectory and the SNR value selected. The results illustrate the capability of SOFMAT to reconstruct complex fiber tract configurations. The ability of SOFMAT to detect fiber paths in low anisotropy regions, which physiologically may correspond to either grey matter or pathology (abnormality) and uncertainty areas in real data, is an advantage of the method for future studies.
\end{abstract}

\section{Introduction}

Diffusion tensor magnetic resonance imaging (DTMRI, also called DTI) is a fundamental technique that allows in vivo structural brain imaging by white matter estimation $[1,2]$. Differing from the weighted MR images, DTI provides directional information that could be used to compute nerve pathways. The modality is unique in its ability to provide in vivo anatomical fiber tract information noninvasively. However, the accurate estimation of white matter fibers is highly dependent on the tractography algorithm used. DTI is advantageous in clinical neuroscience, for quantitative comparison of specific white matter pathways in disease, in guided interventions, for the exploration of the normal brain anatomy [3]. Tractography however, should be used with care because of the limitations of the technique. A complete and validated neural fiber map of human brain is still not available in the literature, which makes the adequate verification of the postprocessing a challenging and a critical task.
An important drawback in the determination of the fiber paths for tractography purposes occurs in uncertainty regions where at least two fiber paths intersect. This study proposes an artificial neural network approach named SOFMAT based on self-organizing feature mapping (SOFM or SOM) to define the fiber tracts based on their diffusivity and to clarify, especially, the fiber tracts in these uncertainty regions $[4,5]$. The locally computed diffusion tensors shape the randomly distributed artificial neuronal topology.

The developed novel SOM-based tractography approach self-organizing feature mapping tractography (SOFMAT) is based on unsupervised learning method, which is used in the training of artificial neural networks (ANNs) $[4,5]$. Unsupervised learning is preferred for the fact that we do not have a reliable training set either for the pathological or for the normal human brain.

Especially in studies dealing with complex data, ANN is very useful and preferable. The use of ANN has a wide range, such as analyzing seismic signals [6], wind speed 
forecasting [7], feature prediction in urban traffic flow [8], in sludge bulking [9], and in founding of reference voltage of maximum power point under different atmospheric conditions [10].

In our study, SOM is selected to train the ANN, because $\mathrm{SOM}$ as a classifier demonstrated successful identification of structured topologies in various domains $[4,5]$. Representing a subset of ANNs, SOM is particularly useful in investigating multidimensional topologies. In this study, the topology sought is actually the tracts of localized diffusion eigenvectors, which define the principal diffusivity of the fibers in the DTMR images. The available anatomical atlases depicting nerve tracts have poor resolution capable of distinguishing millions of axons contained in unit imaging voxel. Clinical validation data is hard to come by for the intended clinical utilization. Instead, it is a common practice to employ artificially produced data to evaluate proposed tractography algorithms. Therefore, in this study, a common diffusion tensor resource named phantom images for simulating tractography errors (PISTE) is used for benchmarking the accuracy and acceptability of the proposed approach.

The idea of SOFMAT is to accomplish the fiber pathways by considering each individual voxel's contribution taking into account the neighboring voxels' behavior in the topology. This is achieved by both the competing and the cooperating behavior of SOM nodes (neurons) in forming the topology. The proposed method has been tested on four phantom images from PISTE with various signal to noise (SNR) values. The images represent various levels of complexities involving crossovers, kisses, and direction changes. The results were then compared against well-accepted tractography algorithms reported in the literature (i.e., streamline (SLT) [1] method and Guided Tensor Restore Anatomical Connectivity Tractography (GTRACT) algorithm [11].

Preliminary studies indicate that SOFMAT method gives promising and relatively superior results compared to the traditionally implemented and well-accepted tractography algorithms mentioned above. SOFMAT has the ability to generate tracts in complex fiber structures such as the spiral phantom utilized and represented in this study. The main reason for the development of the SOFMAT method was to tract complex architectures like spiral trajectory where the standard streamline approaches were failing. A typical SLT algorithm follows only a single direction, where SOFMAT evaluates multiple directions regarding the topological neighborhood function [4]. The GTRACT algorithm is affected by noise and crossing fibers as mentioned in the reference [11], where SOFMAT results are relatively superior compared to GTRACT results.

The sections of the paper are organized as follows. In the next section, a brief background work related to the proposed method is introduced including the synthetic data resource utilized for evaluation. Section 3 describes the method of the presented work in detail addressing how SOM is implemented to detect synthetic tracts and how we validated the results. Section 4, results, presents quantitative comparison of the proposed method against the commonly used algorithms. The discussions are given in Section 5.

\section{Background}

2.1. Principles of Diffusion Tensor Analysis. The principles of DTI are based on the Stejskal-Tanner imaging sequence [1]. Physically, the diffusion tensor estimation can be obtained by taking the arithmetic average of the diffusion images in all possible directions [1]. The $3 \times 3$ symmetric diffusion tensor $D$ is calculated from a set of these diffusion weighted images for each pixel as in the following [1].

$$
S_{i}=S_{0} e^{-b \hat{g}_{i}^{T} D \widehat{g}_{i}}
$$

where

$$
\begin{aligned}
& S_{i} \text { is the signal received with the } i \text { th diffusion gradient } \\
& \text { pulse, where } i=1 \text { to } N,(N=6 \text { typically), } \\
& S_{0} \text { is the signal received without the diffusion gradient } \\
& \text { pulse, } \\
& b \text { is the diffusion weighting factor, } \\
& \left|g_{i}\right| \text { is the strength of the } i \text { th diffusion gradient pulse, } \\
& \text { and } \\
& \widehat{g}_{i} \text { is the } i \text { th diffusion gradient vector. }
\end{aligned}
$$

The diagonal and off-diagonal elements of $3 \times 3 D$ can be displayed as an image. The 6 diagonal and off-diagonal elements' detection is only possible with at least 6 diffusion weighted images. These images with diffusion gradient are required to detect diffusion in all directions. The required 6 independent elements of $D$ are achieved by applying diffusion gradients $\hat{g}_{i}$ along at least six noncollinear, noncoplanar directions.

Principal component analysis (PCA) is used to perform the diffusion tensor analysis and compression. The diagonalization of the diffusion tensor as in (2) results in a set of three eigenvalues $\lambda_{1}>\lambda_{2}>\lambda_{3}$ associated with the three eigenvectors $\vec{e}_{1}, \vec{e}_{2}$, and $\vec{e}_{3}$ corresponding to the principal diffusion vectors for each voxel under the study $[1,2,12]$. The eigenvectors, $\vec{e}_{i}$, for a voxel $x$ can be computed as

$$
\begin{gathered}
D_{x} \vec{e}_{i}=\lambda_{i} \vec{e}_{i}, \quad(i=1,2,3), \\
\left|D_{x}-\lambda I\right|=0
\end{gathered}
$$

where $D$ is the diffusion tensor (1) of the standardized data and $I$ is the identity matrix. The eigensystem calculation of the analyzed image data provides information about the diffusion distribution throughout the investigated image. The first principal component $\lambda_{1}$ shows the dominant diffusivity direction. The second and third principal components $\lambda_{2}$ and $\lambda_{3}$ provide information of the intermediate and smallest principal diffusivity, respectively. In diffusion tensor literature, tracking methods rely mainly on the dominant principal diffusivity $\lambda_{1}$. The assumption is that the fibers' orientation is along the principal diffusivity $[2,13-15]$.

2.2. PISTE: DTI Artificial Data. In an attempt to identify nerve fiber trajectories, several DTI based tractography techniques have been proposed to propagate diffusion tensor 
fields. Since it is difficult to validate the findings of a tractography method on brain images, artificially produced validated phantom images are used for benchmarking. One such commonly utilized dataset in DT-MRI tractography literature is called "phantom images for simulating tractography errors" (PISTE). PISTE comprises a set of simulated fiber trajectories designed for testing, validating, and comparing tractography algorithms allowing the investigation of various geometries like linear, linear break, orthogonal crossing, and spiral [16]. Here, the linear trajectory is defined as a straight-forward linear tract, where the so called linear break trajectory of PISTE has a complete break at the tract [16]. As will be explained in detail in Section 4, orthogonal crossing is an example of intersecting fiber structures. This PISTE trajectory is a crossing sample of two fibers intersecting each other at a right angle. Each of these trajectory sets contains a T2 weighted image, with 6 elements of the diffusion tensor achieved by application of 30 different diffusion directions $[14,15]$, and an image of the corresponding eigensystem [16]. The tensor images of different geometries were fed into the proposed tractography system testing for varying SNR levels of 5, 15, and 30 as well as the noise-free condition. The PISTE images used in this study correspond to MR images acquired with $\mathrm{TE}=90 \mathrm{~ms}$, diffusion tract having a T2 of $65 \mathrm{~ms}$, and the background with a T2 of $95 \mathrm{~ms} .30$ diffusion directions were represented in 16 slices of $150 \times 150$ images. The diffusion directions are obtained using an algorithm analog to electrostatic repulsion $[14,15]$.

The DT images are generated on the investigated trajectory with a decreasing anisotropy along the length of the tract, which is overlaid on a homogeneous anisotropic background. The data used in this study is available as 32 bit float binary files at http://cubric.psych.cf.ac.uk/commondti/ [16].

\section{Methods}

3.1. SOFMAT: Self-Organizing Feature Mapping Tractography. Self-organizing feature mapping tractography (SOFMAT) is proposed as a tractography algorithm in this study. It is based on self-organizing feature map (SOM), a family in artificial neural networks. The advantage of SOM lies in its ability of mapping high dimensional data into a 1D, 2D, or $3 \mathrm{D}$ data space, subject to a topological ordering constraint $[4,5]$. SOM is able to learn an input pattern in terms of the patterns' regularities and correlations. As will be explained in Section 3.4, the network adapts the output pattern according to its input. One important feature of SOM is that it is able to process noisy data. This makes the learning rule applicable in diffusion tensor fiber tract analysis. Based on this special class of ANN, the proposed SOFMAT algorithm aims to map the brain's diffusion tensor data into fiber paths using an unsupervised learning method. Unsupervised nature of the learning is essential, since the ultimate challenge is to identify the tractography of brain nerve pathways with no apriori anatomical or pathological information.

SOM orders the data into meaningful topologies corresponding to the given input data. SOFMAT uses this ability in terms of retaining the underlying structure of the input space and enabling a mapped match of the investigated imaging space resulting in nerve fiber tracts as an output. The final tractography is the converged state of an artificial neuronal map obtained by the iterative synaptic weight update process $[4,5]$.

SOFMAT, in an attempt to discover nerve fiber tracts, utilizes an artificial neural network learning scheme inspired by the self-organization in a neurobiological system [17]. This is achieved by implementing the characteristics and basics of SOM unsupervised learning methodology onto the DT eigensystem. Here, each neuron's spatial location in the resulting feature map corresponds to a particular topology of the input data. Differing from a classical SOM application, SOFMAT utilizes the orientation information $\left(\lambda_{1}, \lambda_{2}, \lambda_{3}\right.$, $\left.e_{1}, e_{2}, e_{3}\right)$ inherent in the DT images, along with the positional dependency. In other words, SOFMAT enables the analysis of correlated neighboring nodes with respect to both their spatial locations and the direction of their diffusivities. The "diffusivity informed" SOFMAT uses this information as a topological ordering constraint.

The feature mapping model which SOFMAT implements in this study is arranged in a number of $1 D$ lattice, as described by Kohonen [5]. In this topology, each neuron has a set of neighbors which are influenced by the motion of a target neuron defined by a weighted Gaussian distance function, as explained in detail in Section 3.3. The lattices formed as a result can take any arbitrary shape in the $n$-dimensional input space, even though they are nothing more than strings, as is also the case for axons forming a nerve tract. The proposed method benefits from this feature in detecting the topological nerve fiber map.

3.2. Competitive Process in SOFMAT. SOFMAT inherits unsupervised competitive learning from SOM with the following principles $[4,5]$.

(i) The output neurons of the network compete among themselves to be fired, for a given input pattern.

(ii) Only one output neuron is activated at any one time, called the winning neuron.

(iii) The winning output node is processed by the selforganization progressing towards the input pattern $I$, while dragging its neighbors.

(iv) As an outcome of this self-organized competition and cooperation, the topological connectivity in $I$ is maintained and reflected in the output.

(v) The input pattern, $I$, selected randomly is represented as

$$
I=\left[x, y, z, \lambda_{1}, \lambda_{2}, \lambda_{3}\right]^{T} \text {, }
$$

where $x, y$, and $z$ correspond to the three position coordinates and $\lambda_{1}, \lambda_{2}$, and $\lambda_{3}$ are the three eigenvectors of the diffusion tensor computed for the simulated diffusion tensor images.

(vi) The input space pattern $I$ and an output node similarity or distance is determined in relation to the associated synaptic weight vector of each output neuron (node) expressed as [4] in (5). Here similarity match 


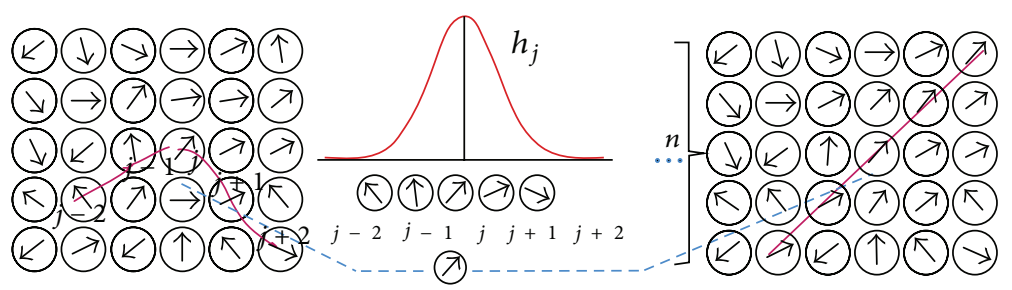

$I(t) \oslash$

(a)

(b)

FIGURE 1: Illustration of the training process. (a) Initial random state of the lattice. The input data vector is displayed here as $I(t)$. Randomly initialized network after a learning step; intermediate stage of self-organization. Best match is assigned as winning node. Updating the weight allows the network to find its best matching nodes in the discrete output space (5). The nodes within the neighborhood $h_{j}$ learn from the winning node. (b) Fully trained network after $n$ iterations: Structured input space.

is reached by identifying the node that best matches the input $I$, and this winning neuron $P(I)$ is found at a time step $t$ by using the minimum-distance Euclidean criterion [4], where $n$ is the total number of neurons in the network:

$$
P(I)=\arg \min _{j}\left\|I(t)-w_{j}\right\|, \quad j=1,2, \ldots, n,
$$

where $w_{j}$ is the weight vector for the $j$ th node as

$$
\vec{w}_{j}=\left[w_{j 1}, w_{j 2}, \ldots, w_{j m}\right]^{T} .
$$

In SOFMAT, $m$ is defined as 6 corresponding to the number of diffusion gradient pulses (1). The first three elements of $w_{j}$ describe the position of $j$ th node, and the last three correspond to the orientation of the vector connecting the $j$ th node to the $j+1$ st node.

SOFMAT identifies the winning neuron by computing a distance function comparing an input pattern $I$ with the synaptic weight vectors, $w_{j}$ for each node (5). The training process is illustrated in Figure 1. First, the weight vectors are mapped randomly onto a two-dimensional lattice. Figure 1(a) represents this initial random state of the lattice. Training of the network gives the closest match to the input data vector $I(t)$ in the node (Figure 1(b)). The nodes within the neighborhood $h$ learn from the winning node (Figure $1(\mathrm{c})$ ). The weight vectors within the neighborhood $h$ learn from the input data vector and get updated. In Figure 1(d), the final, fully trained network is displayed.

3.3. Cooperative Process in SOFMAT. Cooperation in SOM algorithm is also inherited in SOFMAT. The level of cooperation of the neighborhood neurons is decided by the winning neuron (5).

(i) The topological neighborhood $h_{j, i}$ is typically chosen as a Gaussian function [4]:

$$
\begin{gathered}
h_{j, i(x)}(t)=e^{\left(-d_{j, i}^{2} / 2 \sigma^{2}(t)\right)}, \\
\sigma(t)=\sigma_{0} e^{\left(-t / \tau_{1}\right)},
\end{gathered}
$$

where $r$ is the sequential distance and $d_{i j}$ is the Euclidean distance between the winning neuron $P(I)$ and the other neurons $(j)$ in the string and calculated by the sequential distance $r$ of $I$ and $j$ as given in the following:

$$
d_{j, i}^{2}=\|r(j)-r(I)\|^{2}
$$

The width, $\sigma(n)$, of the Gaussian neighborhood function decreases for facilitating convergence at an exponential rate, and the neighborhood shrinks in each iteration. The dependence of $\sigma$ in discrete time $t(t=0,1,2, \ldots)$ in (8) contributes to the convergence of SOM learning algorithm by excluding more nodes from the neighborhood iteratively. $\sigma_{0}$ is the initial value of $\sigma$, and $\tau_{1}$ is the time constant, which are determined by ad hoc methods, influenced by the size of the input space and the number of output nodes as described in [5].

3.4. Adaptive Process in SOFMAT. For a given input pattern $I$, all the neurons in the vicinity of the winning neuron $P(I)$ are updated by a distance coefficient decreasing with the neighboring function, $h_{j, i}$. Note that $h_{j, i}$ is 1 for the winning neuron and decreases exponentially as the nodes gets away from the winning neuron. The iterative learning process is actually representing the adaptation of the weight vectors towards an input pattern $I$ and given as

$$
\vec{w}_{j}(t+1)=\vec{w}_{j}(t)+\eta(t) h_{j, i(I)}(t)\left(I-\vec{w}_{j}(t)\right) \text {. }
$$

Equation (10) gives the computation of the updated weight vector $w_{j}(t+1)$ at time $t+1$, with a time varying learning rate $\eta(t)$, where $w_{j}(t)$ is the synaptic weight vector of neuron $j$ at time $t[4,5]$. As introduced in (4), our input space $I$ consists of the positional elements $x, y$, and $z$ and directional elements $\lambda_{1}, \lambda_{2}$, and $\lambda_{3}$ for each voxel. The SOFMAT is shaped by comparing each input vector $I$, to every node, in the neighborhood (Figure 1). The comparison is based on both position and orientation according to (5). Once the winning neuron is determined, the positions of the nodes are updated according to (10). The directional vectors of the SOFMAT nodes are updated according to the newly formed neuronal topology as in Figure 2. Assuming that nerve 


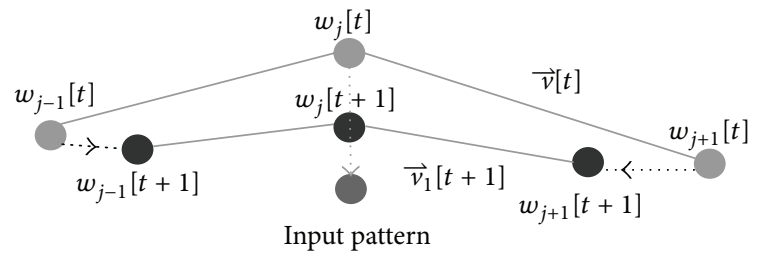

FIGURE 2: The pixel with the weight $w_{j}$ at time $t$, nearest to the input pixel, is winning and is moving towards this input pixel according to (10) as shown with an arrow in the figure. Its neighbors, $w_{j-1}$ and $w_{j+1}$ at time $t$ on the string, are also moving to a lesser extent. The update in the orientation can be recognized through initial weight vector $v[t]$ and updated resulting vector $v[t+1]$. Units close to the winner as well as the winner itself have their weights updated significantly. Weights associated with far away output pixels do not change significantly. The update continues until the sought topology is found and the feature map is consistent.

tracts are formed from multiple axons, we provided multiple strings with an expectation to detect the underlying neuronal pathways. Multiple strings implementation methodology is summarized as follows:

SOFMAT with multiple strings:

(i) initially there are $N_{y}$ strings each of which is made of $N_{x}$ nodes;

(ii) initial position and orientation of each node $(w)$ are randomly initialized;

(iii) for each node of the input pattern, $I$, the winning neuron is computed based on the minimization of the cost function as given in (5). The winning node also determines the winning string;

(iv) once the winning neuron and its string are determined,

(a) a weight update matrix $w_{j}(t)$ is computed for that string using the position information according to (7), (8), and (10),

(b) the weight update matrix is computed for that string for updating the orientation information according to the new position as in Figure 2;

(v) this procedure is repeated from step (iii) until the maximum number of predefined iteration or convergence is reached;

(vi) the converging weight matrix that includes the position and orientation information of the multiple strings is the resulting topology of SOFMAT.

The aim of the implementation is to map the underlying topology of a discrete input space. Initially, the weights are assigned randomly and the SOM pattern is arbitrarily positioned. A starting input node is randomly picked among the inputs for training (Figure 2). The node with the closest reference vector represents the winning neuron $w_{j}(t)$. At the iteration at discrete time $t$, the winning neuron $w_{j}$ moves towards the input pattern (Figure 2), and the two neighboring neurons $w_{j-1}$ and $w_{j+1}$ in its Gaussian neighborhood move in smaller steps. The goal is to train the net until the topology is stable.

For each position update of a node the directional convergence of orientation vector is also achieved.

Following the three processes of the unsupervised learning method, taking into account both the position and the direction of a candidate node, SOFMAT enables the determination of neural fiber tracts having similar diffusivity. The updated neighborhood helps to compute the proper neighbor of each winning neuron, which enables the algorithm to calculate the neural paths with respect to the underlying diffusivity.

3.5. Experimental Methods. The number of inputs is determined by the image dimensions, which is $150 \times 150$ pixels in PISTE. The number of strings and the number of nodes in each string have been changed between 2-80 and 50-3200, respectively, for experimenting the convergence behavior of different PISTE topologies. The learning rate in (10) was set as 0.1 , a typical rate for safe and stable convergence in the expense of slow learning and increased risk of local minima. Each individual PISTE pattern is examined for a number of iterations. For linear and linear break PISTE patterns, 500 iterations were sufficient. For the spiral trajectory the number of iterations was 6000 as expected. By varying iterations, the best match and the determination of the most reliable track is aimed. The more complex the investigated pattern becomes, the more iterations are needed. This is a natural characteristic of a self-organizing network. The main constraint here is the convergence. In each experiment the convergence is checked upon both the position and orientational training results. More detailed explanations and their discussions on experiments and their results are presented in Sections 4 and 5.

The tracking results for SOFMAT are shown on four exemplary synthetic data sets. The PISTE trajectories described in Section 2.2 were selected for the evaluation and comparison purposes when GTRACT and SLT methods were employed in the literature [11]. Both GTRACT and SLT are diffusion tensor fiber tracking suites like SOFMAT. The main difference is that these suites include streamline tracking tools. These fiber tracking methods include a guided tracking tool that integrates apriori information into a streamline algorithm. SOFMAT in contrast enables the tracking by detecting and following the orientation of the weighted neighbors. Especially the spiral trajectory which is known to be problematic for fiber tracking methods is also analyzed with SOFMAT. The reconstructed tracts are represented in the results section, overlaid on the T2 weighted MR images or fractional anisotropy (FA) maps of the reconstructed tract.

\section{Results}

4.1. Experimental Scenarios and Targets. In this study, the linear, linear break, orthogonal crossing, and spiral PISTE data sets each of them with individual FA were examined with SOFMAT. Varying FA values give information about the anisotropy and as a result about the anatomy of the tissue investigated. A change in the FA map shows clues about 


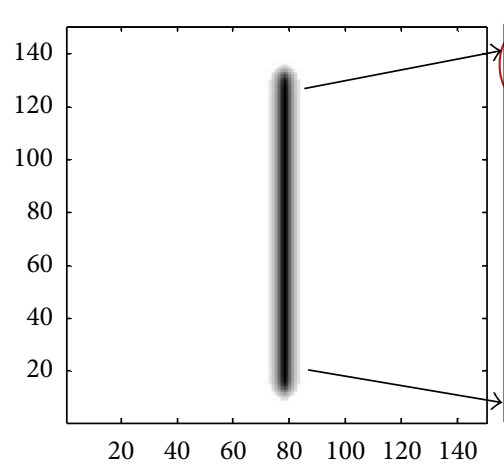

(a)

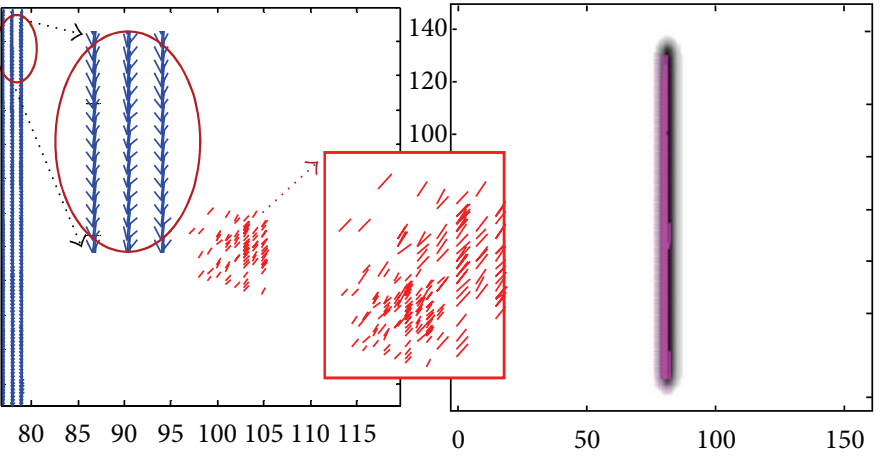

(b)

(c)

FIGURE 3: Linear PISTE trajectory. (a) T2 weighted image; (b) input corresponding to the computed eigenvectors (blue). Initial weights $w_{j 0}$ are seen in red. (c) Pink tracts on the T2 weighted image are SOFMAT's implementation results.

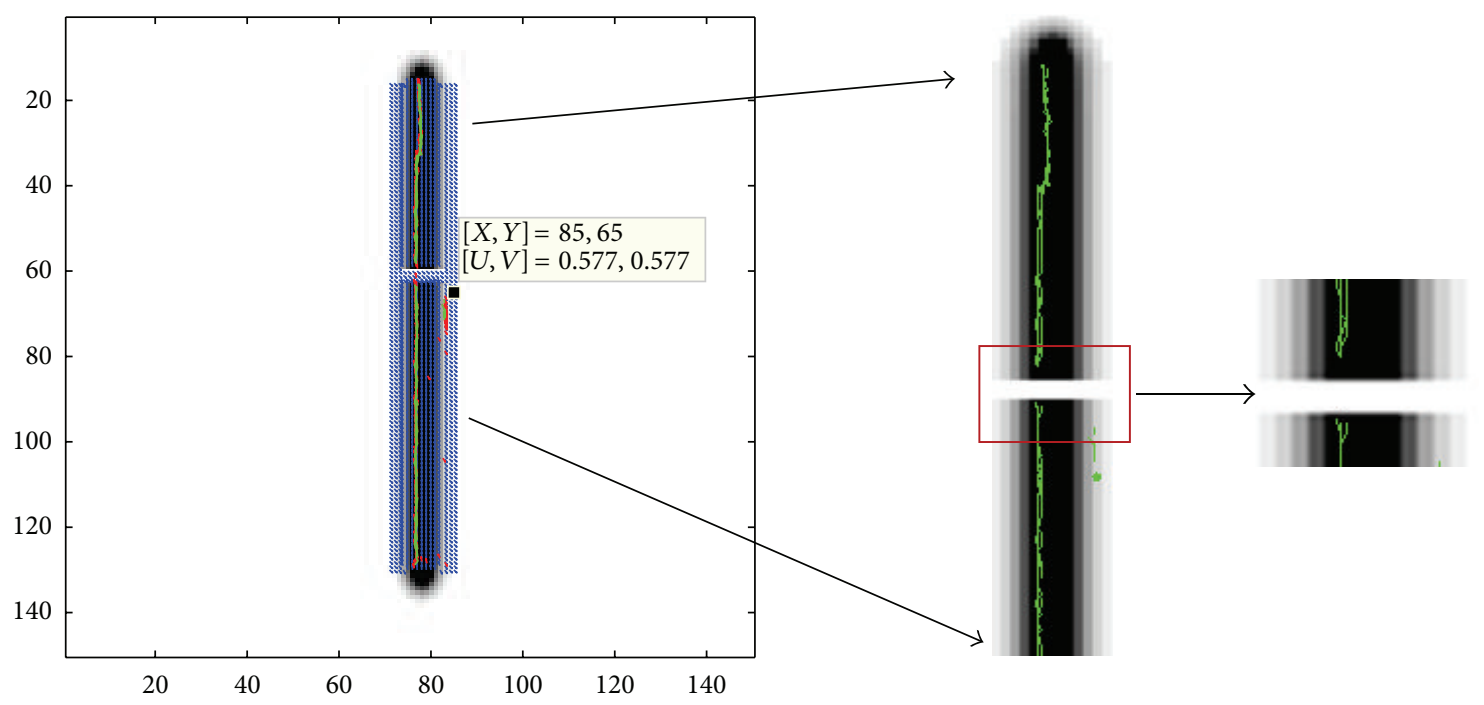

FIGURE 4: Linear break trajectory. Eigenvectors representing the diffusivity are superimposed on T2 image in blue. SOFMAT results with single string trial are seen in green (right). The gap in the middle of the tract is zoomed to give an idea about the implementation result of the algorithm.

the investigated trajectory. In PISTE, images are created on homogeneous anisotropic background, and decreasing anisotropy along tracts is applied. Therefore, the FA maps serve as filters where the routinely applied homogeneous anisotropic background can be extracted from the image. This process also acts as a noise removal highlighting the diffusion pattern. The eigensystem of $D(2)$ is determined by principal component analysis (PCA) $[1,2,12]$, and the principle diffusion direction is interpreted graphically in Figure 3. The entire DT resource is investigated with the proposed SOFMAT method. The search process of the pattern in the selected limits is completed in examining the eigenvectors of each pixel based on the predefined similarity measure. This examined dataset sample might be a whole image data or a single ROI. In this study, the trajectories are not separated into ROIs, they are examined on whole. The details of the investigated geometries are as follows.

Linear Trajectory with and without Break. The linear trajectory is a straight-forward linear tract (Figure 3(a)). Its background is homogenously anisotropic. One has a complete break at the tract (Figure 4). Along the length of the tract, its FA is linearly decreasing. The reconstructed tracts are plotted over the FA map of the investigated linear fiber trajectory (Figure 3). SOFMAT resolves the break on the linear break trajectory (Figures 4 and 5), and it successfully calculates and reconstructs the tracts on the investigated fiber and not on the break (Figure 5).

Orthogonal Crossing Trajectory. This PISTE trajectory is a crossing sample of two fibers intersecting each other at a right angle. The FA values of each of these two orthogonal linear tracts have a slight difference as represented in the orthogonal elements in diffusion tensor image (Figure 6). In this simulated data, the difference on fibers with higher and lower FA values is observed. The SOFMAT results are evaluated with respect to their cost functions by means of spatial distance and angular similarity (Figure 8). SOFMAT reconstruction is displayed in Figure 7. Here, the network has converged with $40 \times 20$ nodes in 500 iterations. 


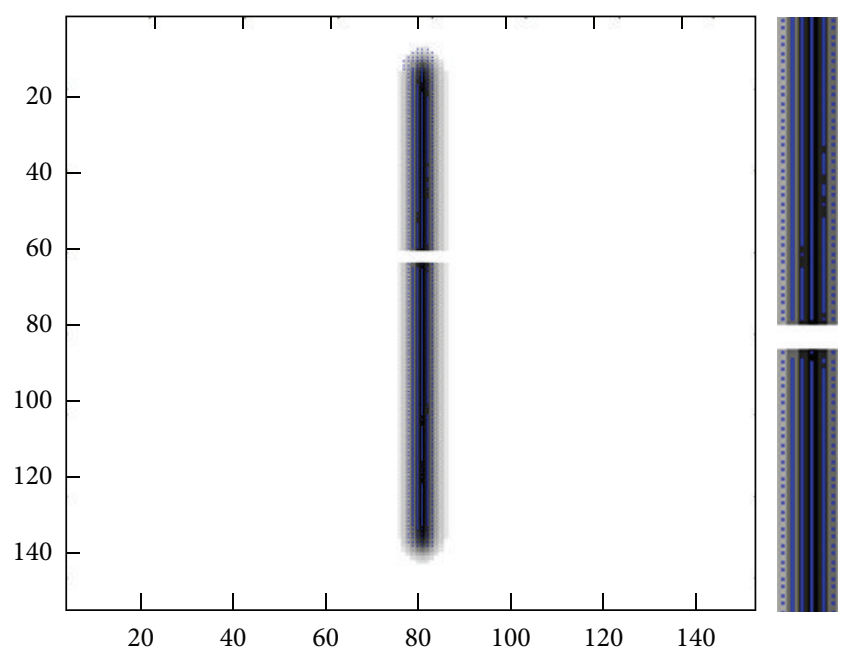

FIGURE 5: Linear break trajectory with multiple strings. SOFMAT results are seen in blue. The trajectory and the gap in the middle of it are determined by SOFMAT as represented.

TABLE 1: The tracking errors (in $\mathrm{mm}$ ) of the three tracking tools for linear trajectory.

\begin{tabular}{llcc}
\hline & SLT & GTRACT & SOFMAT \\
\hline SNR $=30$ & 0.63 & 0.60 & 0.46 \\
SNR $=15$ & 0.90 & 0.65 & 0.48 \\
SNR $=5$ & 1.40 & 0.70 & 0.56 \\
\hline
\end{tabular}

Spiral Trajectory. The spiral trajectory of PISTE is an in-plane spiral tract overlaid on a homogenous isotropic background. It has a high FA value. Here, not only the noise-free sample is investigated by SOFMAT but also the curvy trajectory is examined for SNR values of 30 and 15. Spiral tracts are especially problematic for streamline tractography $[1,13]$ because curvy trajectory cannot be reconstructed accurately by this method. So it is not able to follow the relatively high curvature of the fiber. SOFMAT, however, has the ability to follow the curvature (Table 3). The reconstruction results are presented in Figure 9.

The proposed SOFMAT algorithm is compared with the two well-known fiber tracking suits, GTRACT [11] and SLT [1] using PISTE dataset as a benchmark. The tracking results for the three algorithms were compared qualitatively and quantitatively. As indicated in the reference papers $[18,19]$, the error in fiber tracking is proportional to the square root of the distance along the track. The evaluation of GTRACT algorithm [11] has been performed regarding the predefined error definition as in $[18,19]$. Also in this work, the error is computed based on distance measure as in the definition in the literature. In GTRACT [11], the tracking error was accessed on the linear trajectory and the orthogonal crossing trajectory. For that reason, error of SOFMAT is computed for the two phantom trajectories, the linear and the orthogonal crossing, and computation results are presented in Tables 1 and 2. Results indicate that SOFMAT gives promising and relatively superior results compared to SLT and GTRACT
TABLE 2: The tracking errors (in $\mathrm{mm}$ ) of the three tracking tools for orthogonal crossing trajectory.

\begin{tabular}{lccc}
\hline & SLT & GTRACT & SOFMAT \\
\hline SNR $=30$ & 0.675 & 0.65 & 0.46 \\
SNR $=15$ & 0.875 & 0.66 & 0.48 \\
SNR $=5$ & 1.45 & 0.70 & 0.65 \\
\hline
\end{tabular}

TABLE 3: The mean tracking errors (in $\mathrm{mm}$ ) of SOFMAT reconstruction of spiral trajectory.

\begin{tabular}{lcc}
\hline & \multicolumn{2}{c}{ SOFMAT } \\
\hline Noise free & 0.699 & Angular \\
SNR $=30$ & 0.743 & 0.1151 \\
SNR $=15$ & 1.611 & 0.1127 \\
SNR $=5$ & 6.298 & 0.3416 \\
\hline
\end{tabular}

results. SOFMAT has the ability to generate complex tracts. Aside from the linear, linear break, and orthocrossing trajectories mentioned in [11], spiral trajectory is also investigated in this study (Figure 9; Table 3).

The results for all the three tracking tools are represented in Tables 1 and 2 for all the existing linear and orthogonal PISTE trajectories, respectively. The mean tracking errors in SLT, GTRACT, and SOFMAT for linear PISTE trajectory with an SNR of 30 are $0.63 \mathrm{~mm}, 0.60 \mathrm{~mm}$, and $0.46 \mathrm{~mm}$, respectively (Table 1 ). With an SNR of 5 , again for the linear trajectory, the tracking errors for SLT, GTRACT, and SOFMAT are $1.40 \mathrm{~mm}, 0.70 \mathrm{~mm}$, and $0.56 \mathrm{~mm}$, respectively (Table 1). The mean tracking errors in SLT, GTRACT, and SOFMAT for orthogonal crossing phantom with an SNR of 30 are $0.675 \mathrm{~mm}, 0.65 \mathrm{~mm}$, and $0.46 \mathrm{~mm}$, respectively (Table 2). The mean tracking error in SLT and GTRACT for orthogonal crossing phantom $(\mathrm{SNR}=5)$ are 1.45 and $0.7 \mathrm{~mm}$, respectively. SOFMAT's tracking error for SNR $=5$ for orthocrossing phantom is $0.65 \mathrm{~mm}$ (Table 2). Here, the SOFMAT parameters $N_{x}$ and $N_{y}$ are in each experiment 80 and 40, respectively. The parameters are assigned based on the network's convergence status. The updated network parameters are given in detail in Table 6 .

To observe the effectiveness of the ANN based algorithm, the convergence of the cost function is detected as SOFMAT weights are stabilized. The SOFMAT tracking results of an uncertainty region, namely, an orthocrossing trajectory, are also presented in Figure 7.

In all examinations, the input pattern is the $\mathrm{T} 2$ weighted image of spiral trajectory with input matrix size of $150 \times 150$. In order to compare SOFMAT's results, the network in all of the three cases has 50 strings, where the number of iterations is 6000 and unique in all three exams.

For each of the investigated trajectory, the network's parameters are methodically and carefully determined. The determination of parameters effect the phases of the network and its ability to converge safe and stably. As mentioned previously, each individual PISTE pattern is examined for a number of iterations. The aim of varying iterations is to find 

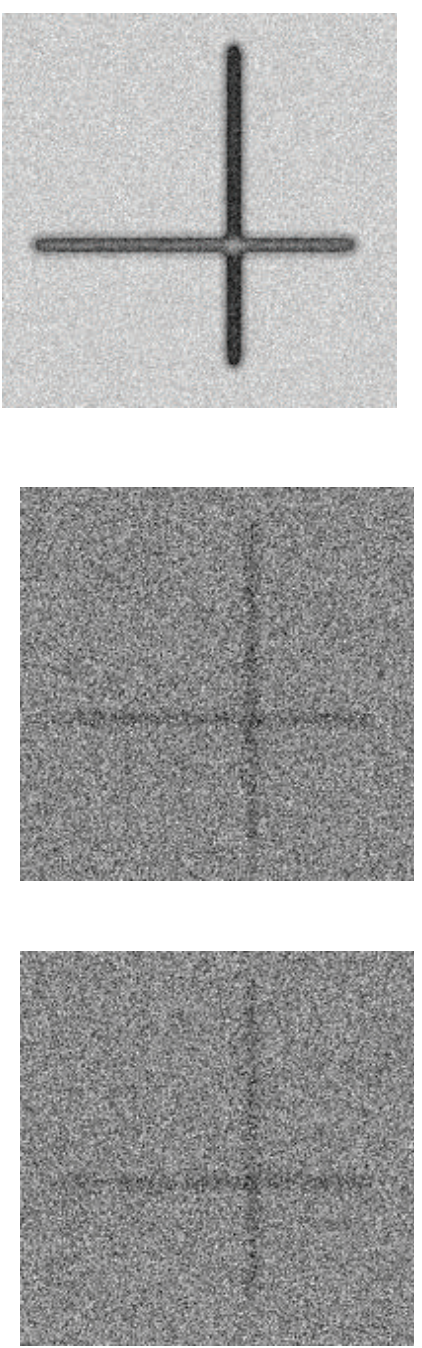

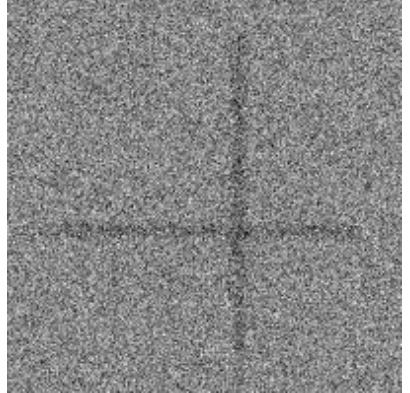

(a)

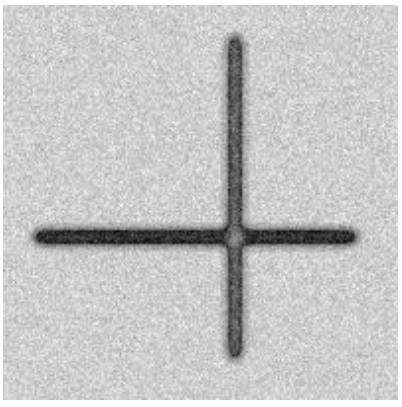

(b)

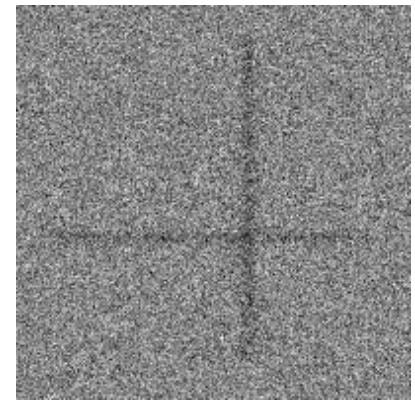

(c)
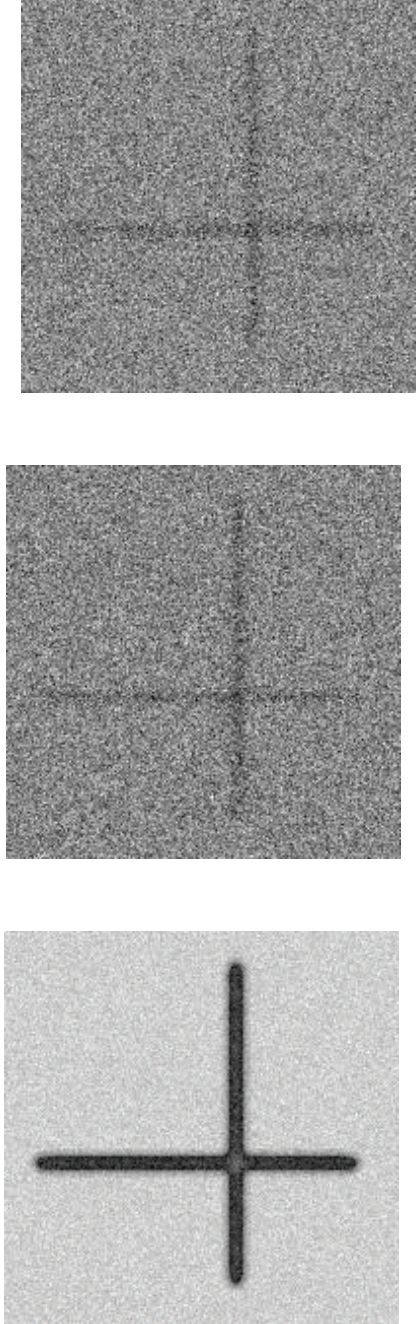

FIGURE 6: Diffusion tensor representation of the orthogonal PISTE trajectory. The different diffusivities for this geometry are seen on the diagonal images. Upper left: $D x x$-the anisotropy of $D$ in $x$ direction and in the middle: $D y y$-the anisotropy of $D$ in $y$ direction.

the best match and so to determine the most reliable tract. The more the investigated pattern gets complex, iteration number increases. This also explains why the spiral trajectory's iteration number $(=6000)$ was the highest among all the trajectories. This is a natural characteristic of a self-organizing network.

In each experiment, the convergence is checked upon both the position and orientational training results. Here, the orthogonal crossing trajectory with $150 \times 150$ original input size is selected as a sample. In Tables 4-6, various network parameter selections and their results are represented. First, a network with 2 strings created from 50 to 200 nodes is analyzed. The mean spatial distance between the known values of the input pattern and those calculated with SOFMAT as output vary from 5.7429 to 1.819 pixels. In all of these cases, the iteration number is kept constant with 500 steps. As expected, with increased number of strings, the trajectory is more precisely determined (Table 6).
TABLE 4: The validation results of the SOFMAT implementation. The orthogonal crossing trajectory is selected as sample. Here, analysis results for 2-string case are shown.

\begin{tabular}{lcc}
\hline $\begin{array}{l}\text { Node } \times \text { string } \\
N_{x} \times N_{y}\end{array}$ & Mean spatial distance & Angular norm \\
\hline $50 \times 2$ & 5.7429 & 0.1206 \\
$100 \times 2$ & 3.5785 & 0.0999 \\
$150 \times 2$ & 2.2843 & 0.1003 \\
$200 \times 2$ & 1.8195 & 0.0965 \\
\hline
\end{tabular}

\section{Discussion}

Several studies aim to investigate the synchronization, information transmission, and signal sensitivity in concept of theoretical neuroscience using neural networks [20-23]. There are studies which concentrate on the synchronization on 


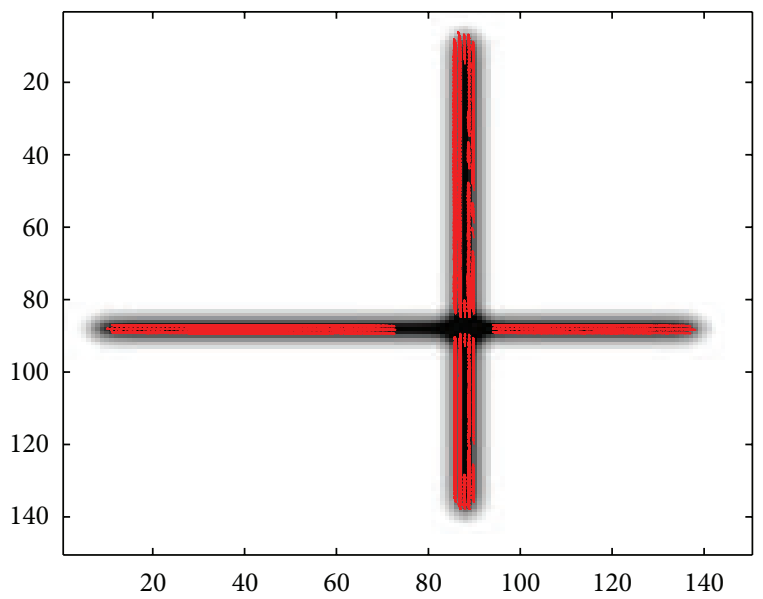

Figure 7: The SOFMAT result superimposed on T2 images for orthogonal crossing trajectory. The tracts are defined along the paths through the total trajectory. Both of the orthogonal tracts are reconstructed completely.

TABLE 5: The validation results of the SOFMAT implementation for orthogonal crossing. A wider network for orthogonal crossing trajectory is investigated and represented.

\begin{tabular}{lcc}
\hline $\begin{array}{l}\text { Node } \times \text { string } \\
N_{x} \times N_{y}\end{array}$ & Mean spatial distance & Angular norm \\
\hline $20 \times 20$ & 8.17 & 0.0923 \\
$25 \times 20$ & 3.3412 & 0.0943 \\
$30 \times 20$ & 2.8286 & 0.0883 \\
$40 \times 20$ & 2.1506 & 0.0910 \\
$60 \times 20$ & 1.7862 & 0.0917 \\
\hline
\end{tabular}

TABLE 6: The validation results of the SOFMAT implementation for orthogonal crossing with updated network parameters.

\begin{tabular}{lcc}
\hline $\begin{array}{l}\text { Node } \times \text { string } \\
N_{x} \times N_{y}\end{array}$ & Mean spatial distance & Angular norm \\
\hline $20 \times 40$ & 1.49455 & 0.0996 \\
$40 \times 40$ & 1.1065 & 0.0971 \\
$60 \times 40$ & 1.0391 & 0.0969 \\
$80 \times 40$ & 0.8346 & 0.0885 \\
$100 \times 40$ & 0.6577 & 0.0330 \\
$150 \times 40$ & 0.8480 & 0.0890 \\
$200 \times 40$ & 0.4263 & 0.0415 \\
$250 \times 40$ & 0.3729 & 0.0436 \\
$300 \times 40$ & 0.3603 & 0.0504 \\
\hline
\end{tabular}

scale-free neuronal networks with phase-repulsive coupling and delay [20-23]. These studies focus on the dependency of synchronization transitions on the information transmission delay over scale-free neuronal networks with attractive or repulsive coupling [24]. Other than biological neuronal studies, our study aims to explore the structural fiber tractography to define the brain fiber paths properly using artificial neural networks. To analyze the complex dynamics of the biological neuronal networks such as excitability delay and complex topologies, it is adequate to develop additional theoretical and experimental studies.

Unlike the functional magnetic resonance imaging (fMRI), DTI highlights the anatomical connectivity patterns of the brain and does not include any information about the function of the brain activity directly. Generally, clinical DTI studies measure the data voxel wise where the size of each voxel is on the order of millimeters. But it's well-known that there are millions of fibers passing through each image voxel. Thus, the spatiotemporal dynamics within a voxel can be explored by using the Hudgkin-Huxley neuronal networks $[24,25]$. In the concept of our paper, the anatomical relationship and connectivity are tried to be estimated among the voxels. The mathematical model that is proposed in [24] can be modified and optimized for the voxel DTI data to deduce the anatomical connectivity in the brain. It may hold significant value for future brain imaging studies.

Our study proposes an artificial neural network approach named SOFMAT based on self-organizing feature mapping to generate simulated tracts based on their diffusivity and to clarify, especially, the fiber tracts in complex fiber architectures. The artificial neuronal topology based on unsupervised learning method is used as a classifier to identify the structured simulated topologies.

Mapping the brain's white matter noninvasively is possible through proper analysis of DTMR images. The algorithms proposed for fiber mapping and fiber tractography are to be examined by synthetically simulated datasets for accurate validation. In this study, a common synthetic DT dataset, namely, PISTE, which is specially generated for verification purposes of DT and tractography algorithms, is used for verification and validation. One of the main constraints in the accuracy of the mapping results is the determination of intersecting fiber tracts in uncertainty regions. In DTI literature these intersecting regions generate a critical tracking problem. Providing a solution for identification of the orientations of the brain fibers in these uncertainty regions in diffusion tensor analysis is of great importance [26, 27]. Methods and updates are to be researched to define these uncertainty regions. Streamline tractography (SLT) is recalled as an accepted basis method for diffusion tensor tractography (DTT). For that reason, SLT is one of the algorithms selected for comparison with SOFMAT (Tables 1 and 2). Secondary, the DTT algorithm chosen for evaluation is the GTRACT software implementation. The SLT uses the principle eigenvector, $e_{1}$, to compute Euler's method approximation to the parameterized tract $[1,13]$.

In this study, we proposed a tracking tool for detecting real brain fibers later as a future study according to unsupervised learning method SOFM. The main idea of SOFMAT is to track the complex fibers according to unsupervised learning while keeping the structural information of the underlying tissue. The methodology is applied and examined firstly on computer simulated trajectories PISTE for verification and validation of the algorithm.

The proposed fiber reconstruction method SOFMAT clarifies the diffusivities in the previously mentioned uncertainty regions (Figures 3, 5, and 9). Quantitative results are 


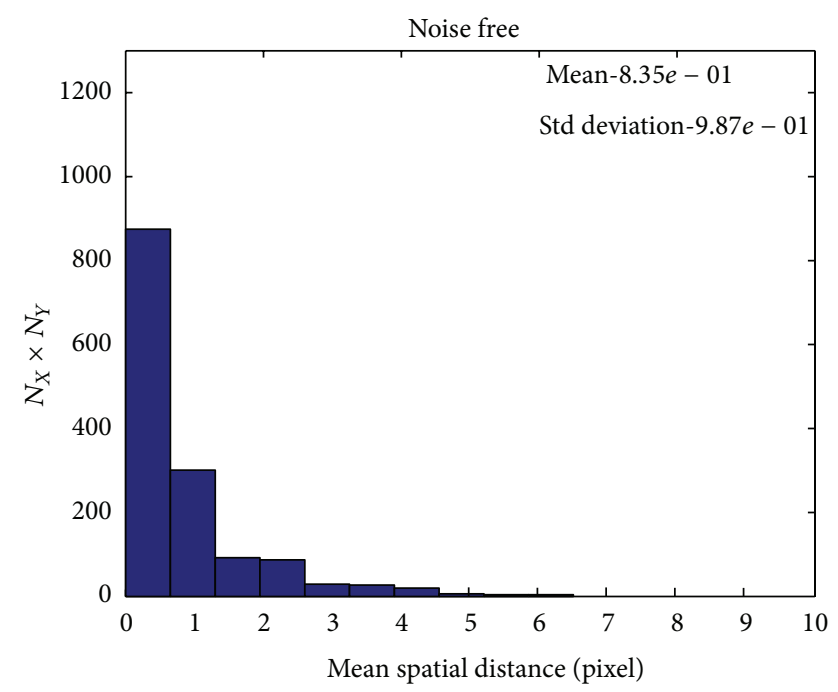

(a)

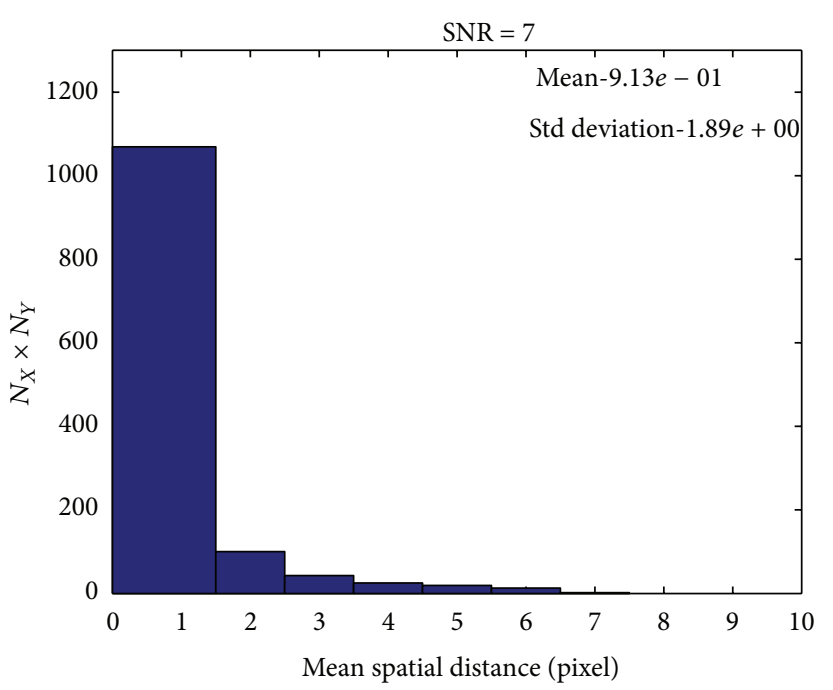

(b)

FIGURE 8: The SOFMAT results are investigated, and the determined neighborhood's coordinates and their related calculated eigenvectors are evaluated. The histogram shows the similarity of the original input and the SOFMAT's reconstructed tract for orthocrossing trajectory: noise free (a) and SNR = 5 (b). The angular cost function results inform that the input and the output are nearly the same. Here, the input pattern is $150 \times 150$, where in both cases noise free and $\mathrm{SNR}=5$, the parameters $N_{x}$ and $N_{y}$ are 80 and 40 , respectively.

listed in Tables 1 and 2 with respect to well accepted tractography techniques $[1,11,13]$. These results are relatively superior to the results of SLT and GTRACT. The figures in the result section are represented to give an idea of how (well) the SOFMAT results match the input patterns. The method is tested with varying SNR values and also in low anisotropy regions. Low anisotropy regions are studied more intensely focusing on the problematic crossings on the phantoms. The fractional anisotropy $(\max . \mathrm{FA}=1$ ) represents the degree of anisotropy, in other words the deviation from isotropic diffusion $(\mathrm{FA}=0)$. The grey matter in the brain is nearly isotropic $(0.37>\mathrm{FA}>0.15)$. In uncertainty regions, it is hard to define the direction of the fiber tracts. Therefore, the detection of fibers in regions having low fractional anisotropy is an advantage of the proposed method. Because in real brain studies, aside from grey matter, low anisotropy regions are uncertainty areas or injured regions. Therefore, the ability to reconstruct the fiber structures in low anisotropy regions is an important benefit of SOFMAT. Optimizing the cost function and neuron selection, the algorithm is able to detect small tract changes and curvy trajectories (Figure 9 and Table 3 ).

Fiber tracking in SOFMAT begins by identifying seed voxels to be used as potential starting positions for the reconstructed fibers. Based on the predetermined eigensystem of the sample trajectories, fiber tract is estimated within each voxel regarding to the diffusivity defined by this eigensystem. Here, the knowledge in diffusion literature suggests that the eigensystem defines the diffusivity $[1,13]$. Each node (neuron) in the region of interest (ROI) is considered as part of a potential fiber tract. The computed winning neurons define the possible nerve tract a fiber can follow with respect to both the coordinate and the directional information of the winning neurons. In other words, a winning neuron determines the newly gained voxel to the tract in terms of its coordinate and direction. The estimation follows by evaluating each node's neighboring function in terms of the similarity criteria.

The novel algorithm SOFMAT is being evaluated throughout the study. The validation study performed on PISTE gives promising results, and they have been compared to the well-known SLT method and the GTRACT algorithm. In the literature, as it has been represented in [11] by GTRACT evaluation, the error is investigated along the resulting tract, and quantitative results are given in Tables 1 and 2. SOFMAT is able to handle fiber crossings and also the spiral trajectory. SOFMAT results to highlight to propagation of fibers through the intersecting and curvy regions are represented in Figures 3, 4, 5, 7, and 9. The minimization of the error is successfully managed where the characteristics can be tracked in related cost function of the network. The increasing number of neurons and iterations selected in the SOM implementation results more reliable tractography outputs (Tables 4, 5, and 6).

Investigating samples with both varying noise and different geometry is important for evaluation, because the deviation from the original fiber path is caused mainly by the noise. The relationship between the tracking error to SNR is acceptable in all examinations. Also dependency on the geometry is seen (Figures 3 and 9). Considering that every trajectory in PISTE has a different diffusivity characteristics, it is meaningful that the cost functions representing the fiber determination performance for linear and orthocrossing geometries vary from each other. The SOFMAT algorithm improves the performance of the fiber tracking even in the presence of noise discussed in Tables 1 and 2. SOFMAT also allows tracking branching fibers. In conclusion, SOFMAT is able to describe two or more fiber tracts simultaneously and 

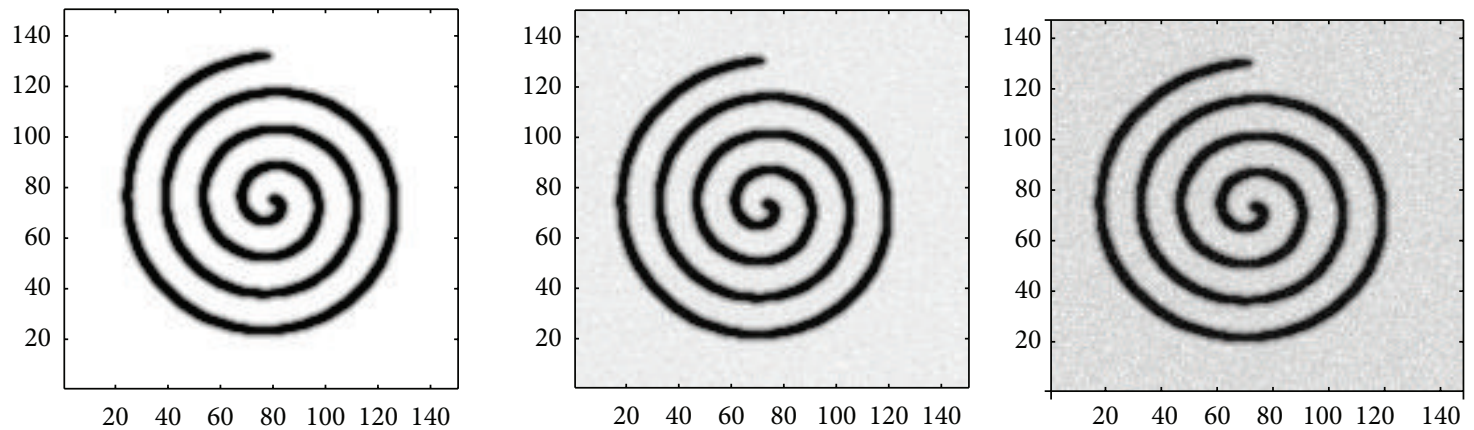

(a)
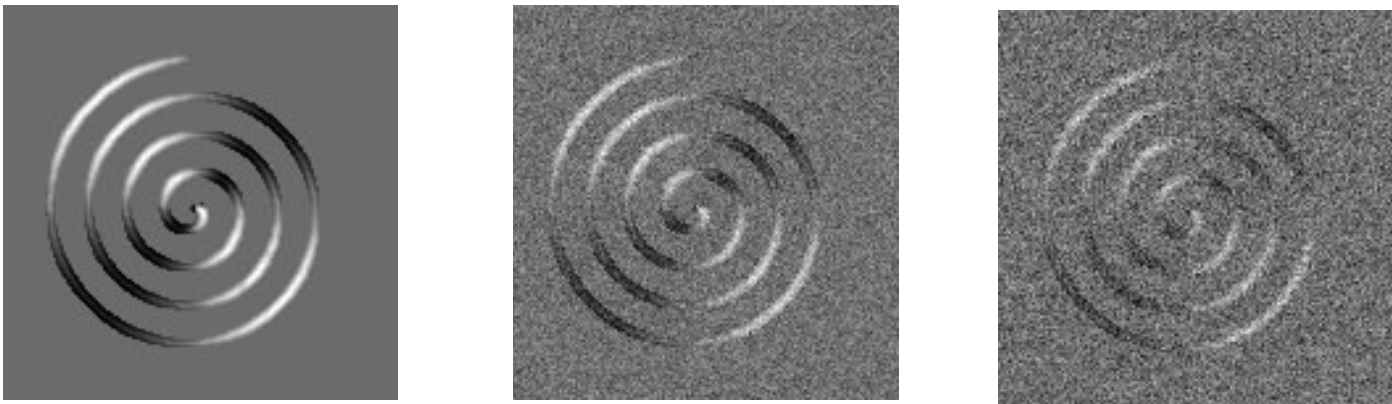

(b)
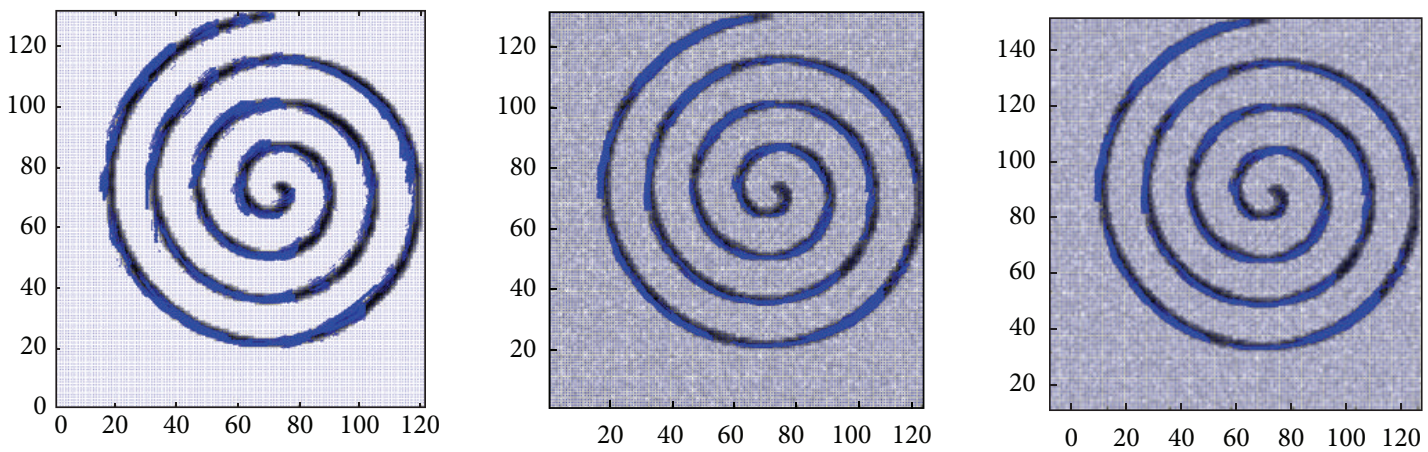

(c)
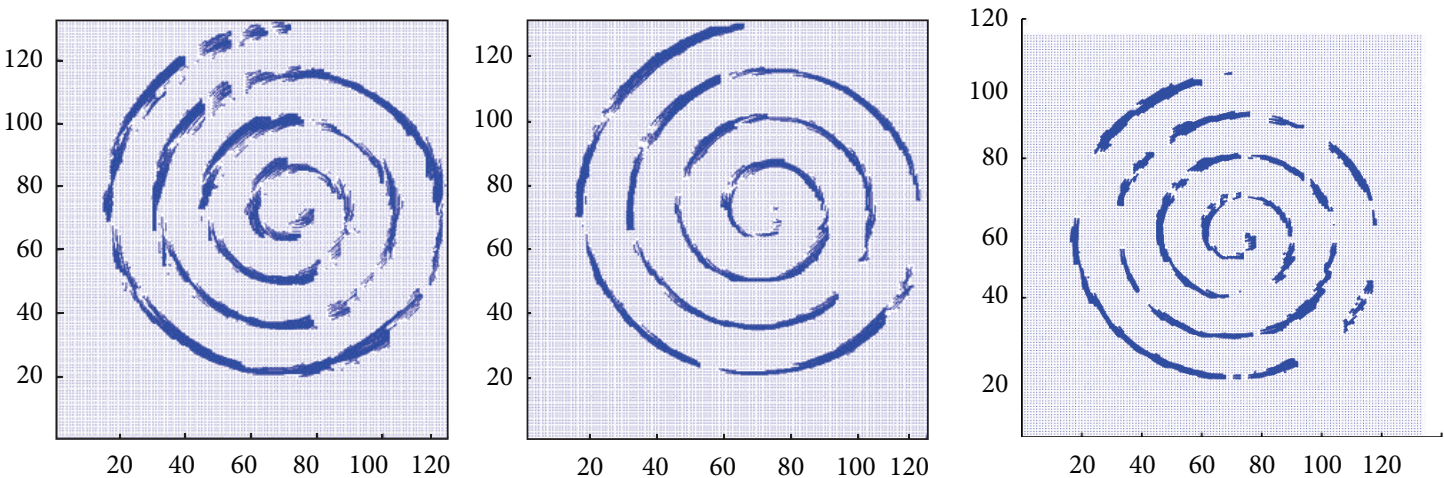

(d)

FIGURE 9: The spiral trajectory results are represented. (a): T2 weighted images; from left to right: noise free T2; SNR = 30; SNR = 15. (b): FA images of each input data. (c): SOFMAT results superimposed on respective T2 weighted images. (d): SOFMAT reconstructions exclusively. 
to reconstruct tracts in uncertainty regions. SOFMAT, on the other hand, does not lose the fiber tractography in relatively high SNRs.

The SOFMAT method gives promising results, compared to the traditionally implemented and well-accepted tractography algorithms mentioned above (Tables 1 and 2). As a future work we plan to implement SOFMAT on real brain.

\section{Conclusion}

This paper represents a novel approach namely self-organizing feature mapping tractography (SOFMAT) for complex fiber tracking purposes in diffusion tensor analysis. The algorithm is based on unsupervised learning in artificial neural networks. As an alternative to the existing methods, SOFMAT is also effective in low anisotropy regions and less affected to noise and curvature complexity than GTRACT and SLT methods. Also, unlike some DTT studies established with PISTE $[28,29]$, the presented SOFMAT study provides performance evaluation other than just visual inspection. The error analysis of the SOFMAT results compared to the existing methods gives improved tract determination and followup. In crossing regions with intersecting fiber distributions and varying SNR values, SOFMAT is able to define the predetermined fiber paths successfully with a standard deviation of $(0.8-1.9) \times 10^{-3}$ depending on the trajectory and the SNR value selected. The results illustrate the capability of SOFMAT to reconstruct complex fiber tract configurations.

\section{Conflict of Interests}

The authors have no conflict of interests to disclose.

\section{Acknowledgment}

This work is supported in part by Bogazici University Scientific Research Project no. 07HX104D.

\section{References}

[1] P. J. Basser, S. Pajevic, C. Pierpaoli, J. Duda, and A. Aldroubi, "In vivo fiber tractography using DT-MRI data," Magnetic Resonance in Medicine, vol. 44, pp. 625-632, 2000.

[2] D. Le Bihan, J. F. Mangin, C. Poupon et al., "Diffusion tensor imaging: concepts and applications," Journal of Magnetic Resonance Imaging, vol. 13, no. 4, pp. 534-546, 2001.

[3] H. Johansen-Berg and T. E. J. Behrens, "Just pretty pictures? What diffusion tractography can add in clinical neuroscience," Current Opinion in Neurology, vol. 19, no. 4, pp. 379-385, 2006.

[4] S. Haykin, Neural Networks: A Comprehensive Foundation, Prentice-Hall, Upper Saddle River, NJ, USA, 2005.

[5] T. Kohonen, Self-Organizing Maps, Springer, Berlin, Germany, 2001.

[6] X. Wang, F. Kang, J. Li, and X. Wang, "Inverse parametric analysis of seismic permanent deformation for earth-rockfill dams using artificial neural networks," Mathematical Problems in Engineering, vol. 2012, Article ID 383749, 19 pages, 2012.
[7] C. Yao, X. Gao, and Y. Yu, "Wind speed forecasting by wavelet neural networks: a comparative study," Mathematical Problems in Engineering, vol. 2013, Article ID 395815, 7 pages, 2013.

[8] C. Ding, W. Wang, X. Wang, and M. Baumann, "A neural network model for driver's lane-changing trajectory prediction in Urban traffic flow," Mathematical Problems in Engineering, vol. 2013, Article ID 967358, 8 pages, 2013.

[9] I. Lou and Y. Zhao, "Sludge bulking prediction using principle component regression and artificial neural network," Mathematical Problems in Engineering, vol. 2012, Article ID 237693, 17 pages, 2012.

[10] F. Sedaghati, A. Nahavandi, M. A. Badamchizadeh, S. Ghaemi, and M. A. Fallah, "PV maximum power-point tracking by using artificial neural network," Mathematical Problems in Engineering, vol. 2012, Article ID 506709, 10 pages, 2012.

[11] P. Cheng, V. A. Magnotta, D. Wu et al., "Evaluation of the GTRACT diffusion tensor tractography algorithm: a validation and reliability study," NeuroImage, vol. 31, no. 3, pp. 1075-1085, 2006.

[12] R. Watts, C. Liston, S. Niogi, and A. M. Uluğ, "Fiber tracking using magnetic resonance diffusion tensor imaging and its applications to human brain development," Mental Retardation and Developmental Disabilities Research Reviews, vol. 9, no. 3, pp. 168-177, 2003.

[13] S. Mori, B. J. Crain, V. P. Chacko, and P. C. M. van Zijl, “Threedimensional tracking of axonal projections in the brain by magnetic resonance imaging," Annals of Neurology, vol. 45, pp. 265269, 1999.

[14] D. K. Jones, M. A. Horsfield, and A. Simmons, "Optimal strategies for measuring diffusion in anisotropic systems by magnetic resonance imaging," Magnetic Resonance in Medicine, vol. 42, pp. 515-525, 1999.

[15] D. K. Jones, A. Simmons, S. C. R. Williams, and M. A. Horsfield, "Non-invasive assessment of axonal fiber connectivity in the human brain via diffusion tensor MRI," Magnetic Resonance in Medicine, vol. 42, pp. 37-41, 1999.

[16] S. C. Deoni and D. K. Jones, "Generation of a common diffusion tensor imaging dataset," in Proceedings of the ISMRM Workshop on Methods for Quantitative Diffusion Methods for Quantitative Diffusion MRI of Human Brain, 2005, http:// cubric.psych.cf.ac.uk/commondti.

[17] M. Spratling and G. M. Hayes, "A self-organising neural network for modelling cortical development," in Proceedings of European Symposium on Artificial Neural Networks (ESANN '98), pp. 333-338, 1998.

[18] N. F. Lori, E. Akbudak, J. S. Shimony et al., "Diffusion tensor fiber tracking of human brain connectivity: aquisition methods, reliability analysis and biological results," NMR in Biomedicine, vol. 15, no. 7-8, pp. 494-515, 2002.

[19] M. Lazar and A. L. Alexander, "White matter tractography using diffusion tensor deflection," Human Brain Mapping, vol. 18, no. 4, pp. 306-321, 2003.

[20] Q. Wang, M. Perc, Z. Duan, and G. Chen, "Synchronization transitions on scale-free neuronal networks due to finite information transmission delays," Physical Review E, vol. 80, no. 2, Article ID 026206, 2009.

[21] Q. Wang, G. Chen, and M. Perc, "Synchronous bursts on scalefree neuronal networks with attractive and repulsive coupling," PLoS ONE, vol. 6, no. 1, Article ID e15851, 2011.

[22] Q. Wang and G. Chen, "Delay-induced intermittent transition of synchronization in neuronal networks with hybrid synapses," Chaos, vol. 21, no. 1, Article ID 013123, 2011. 
[23] Q. Wang, H. Zhang, and G. Chen, "Effect of the heterogeneous neuron and information transmission delay on stochastic resonance of neuronal networks," Chaos, vol. 22, Article ID 043123, 7 pages, 2012.

[24] Q. Wang, M. Perc, Z. Duan, and G. Chen, "Delay-enhanced coherence of spiral waves in noisy Hodgkin-Huxley neuronal networks," Physics Letters A, vol. 372, pp. 5681-5687, 2008.

[25] Q. Wang and Y. Zheng, "Effects of information transmission delay and channel blocking on synchronization in scale-free Hodgkin-Huxley neuronal networks," Acta Mechanica Sinica, vol. 27, no. 6, pp. 1052-1058, 2011.

[26] P. J. Basser, J. Mattiello, and D. LeBihan, "MR diffusion tensor spectroscopy and imaging," Biophysical Journal, vol. 66, no. 1, pp. 259-267, 1994.

[27] D. LeBihan, C. Poupon, A. Amadon, and F. Lethimonnier, "Artifacts and pitfalls in diffusion MRI," Journal of Magnetic Resonance Imaging, vol. 24, pp. 478-488, 2006.

[28] C. Qin, N. Kang, and N. Cao, "Performance evaluation of anisotropic diffusion simulation based tractography on phantom images," in Proceedings of the 45th Annual ACM Southeast Conference (ACMSE '07), pp. 521-522, New York, NY, USA, July 2007.

[29] S. C. Mang, D. Logashenko, D. Gembris, G. Wittum, W. Grodd, and U. Klose, "Diffusion simulation-based fiber tracking using time-of-arrival maps: a comparison with standard methods," Magnetic Resonance Materials in Physics, Biology and Medicine, vol. 23, no. 5-6, pp. 391-398, 2010. 


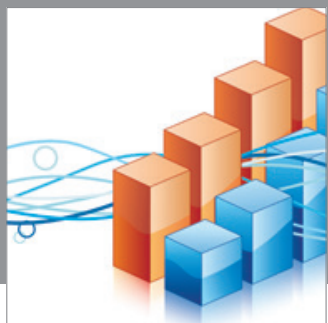

Advances in

Operations Research

mansans

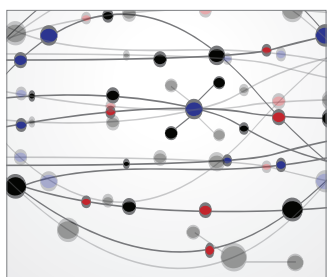

The Scientific World Journal
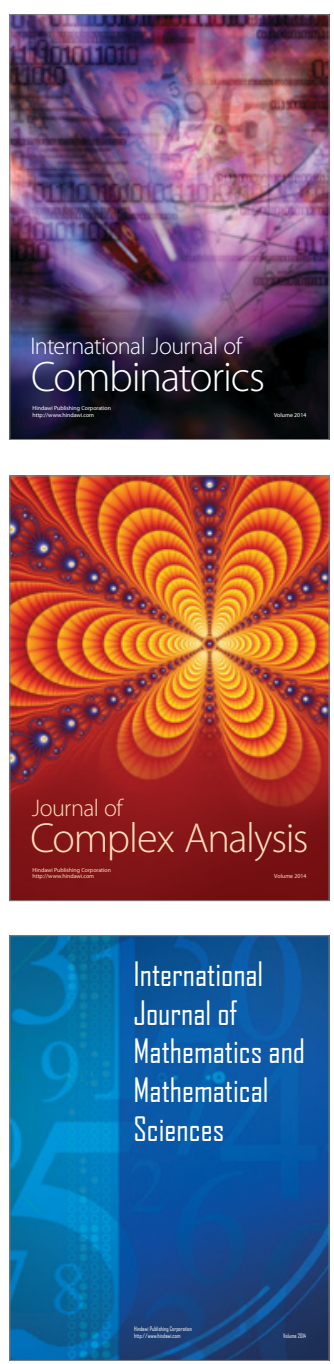
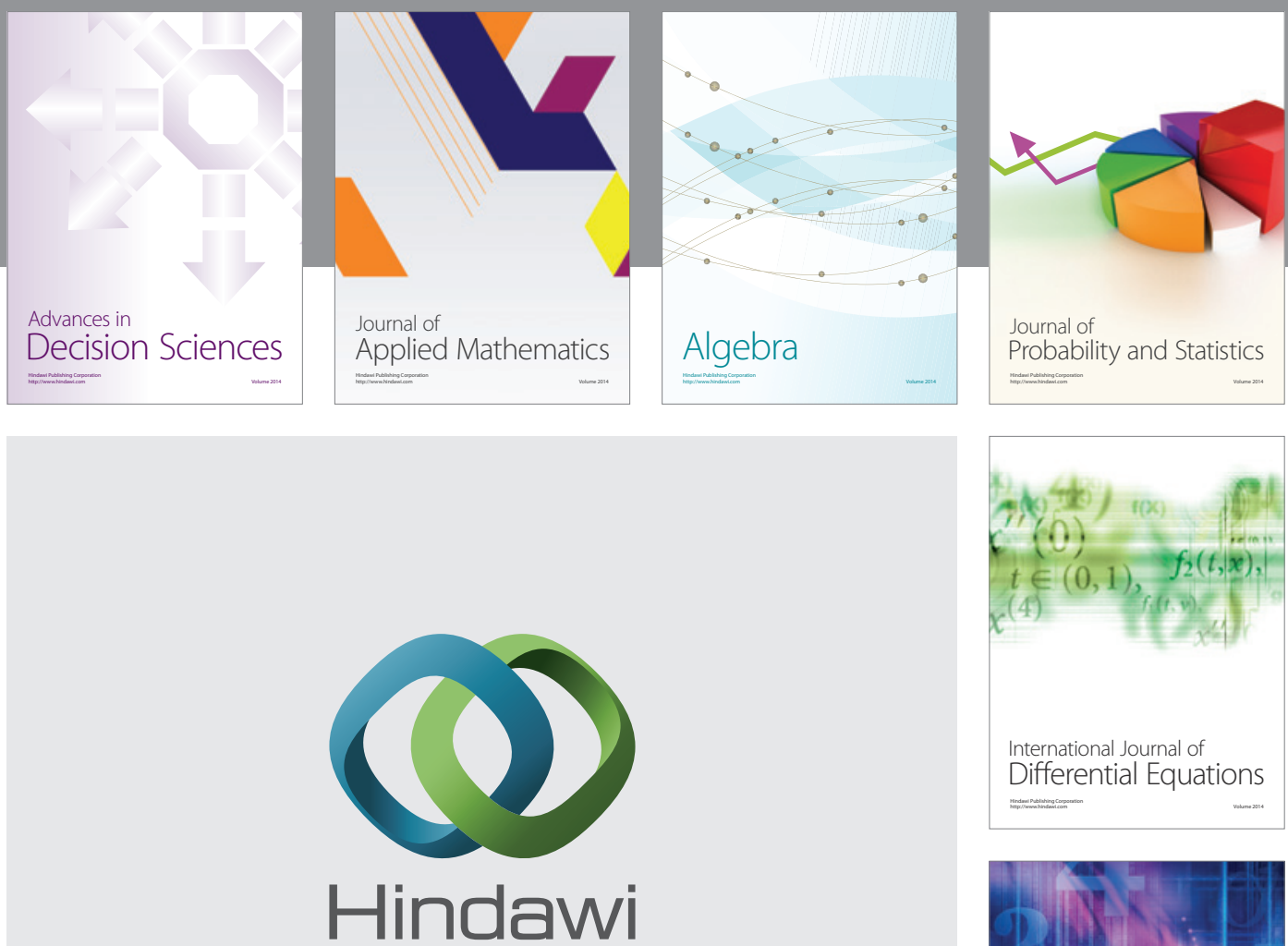

Submit your manuscripts at http://www.hindawi.com
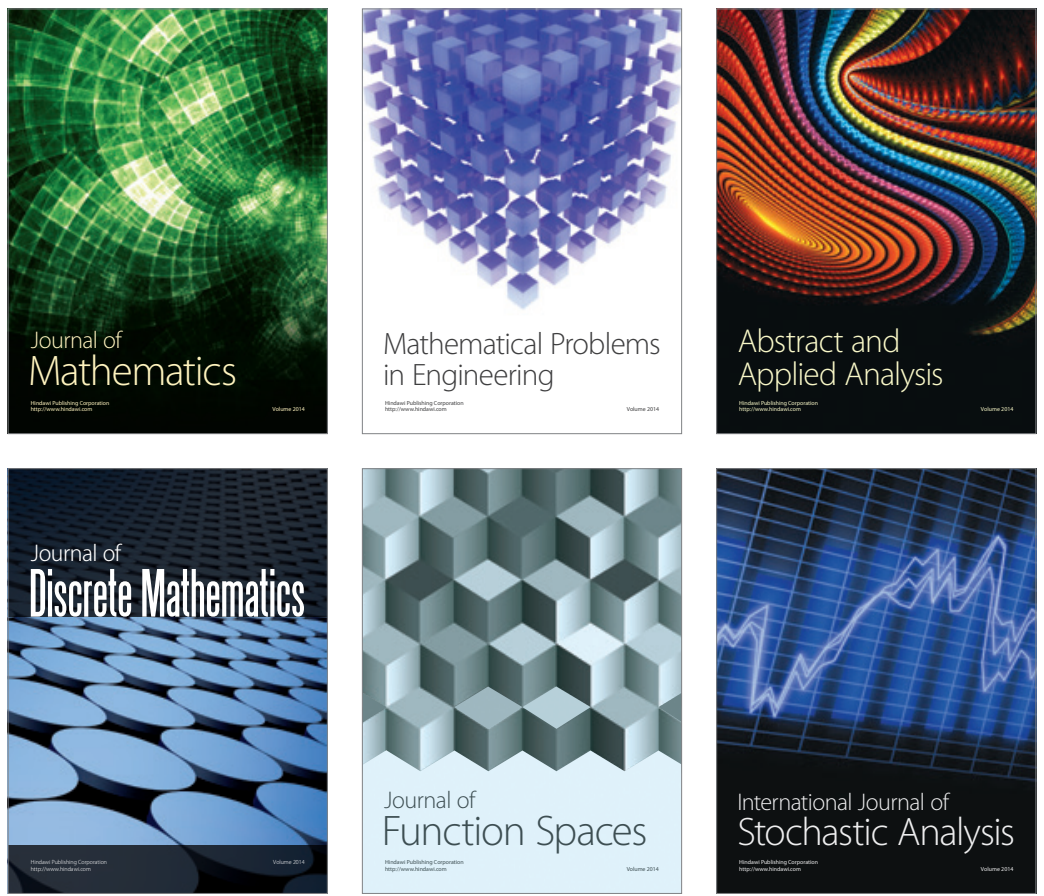

Journal of

Function Spaces

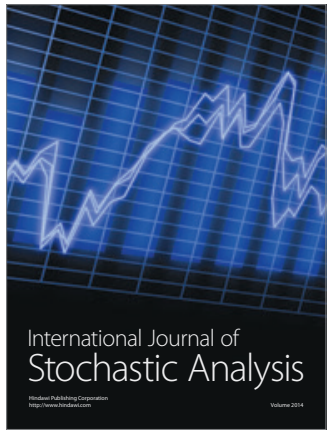

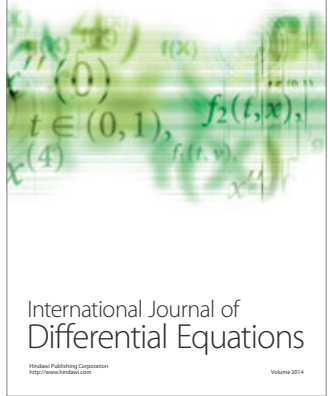
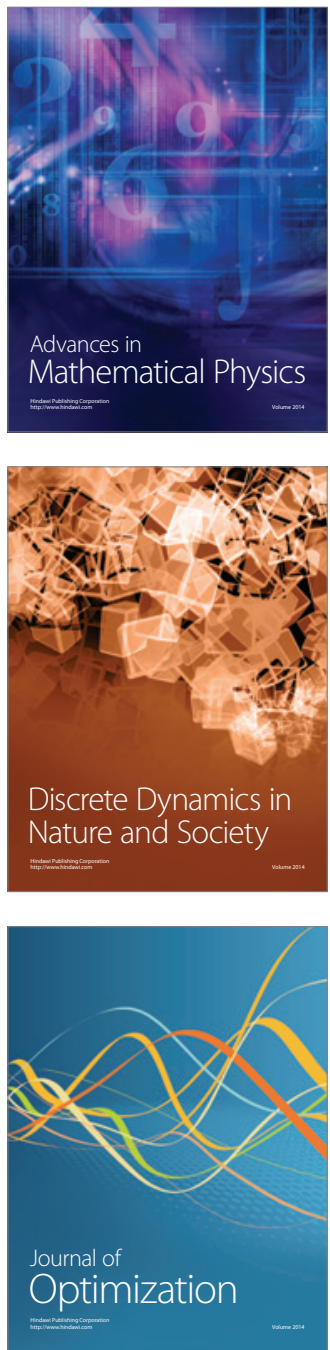